\title{
Challenges for Wireless Power Transfer in Building-Integrated Photovoltaics
}

\begin{abstract}
Building-integrated photovoltaics is steadily entering the market. It allows for solar cells to be an integrated part of the building itself, contrary to installing the photovoltaic modules onto the finished building. Unfortunately, several challenges such as the creation of thermal bridges and moisture intrusion hinder the rapid development of building-integrated photovoltaics into a mainstream mass product. Wireless power transfer systems could solve some of these challenges and contribute to an accelerated use of building-integrated photovoltaic solar cells. In this work, the advantages of wireless power transfer to building-integrated photovoltaics are presented. The different issues and technological challenges are highlighted, and possible solutions are proposed.
\end{abstract}

\section{INTRODUCTION}

The photovoltaic (PV) market worldwide is booming. In general, 'building attached photovoltaics' is applied [1], i.e., the solar panels are added to an already finished building. For example, solar cell modules can be installed above or onto an existing roof or wall. Building integrated photovoltaics (BIPV) differs from this approach: in contrast to building attached photovoltaics, the solar panels of BIPV are an integral part of the building component itself. Their function is not only the production of electricity, but they also serve as a structural element of the building, replacing a conventional building element. In this way, they provide a significant reduced cost in terms of material costs and electricity [2].

BIPV is steadily entering the market, with an installed power capacity of $2.3 \mathrm{GW}$ in 2015 [2]. It has successfully been applied for pitched roofs (solar tiles and shingles), flat and curved roofs (flexible PV laminates), windows and skylights (semitransparent solar cells, often also functioning as shading systems), curtain walls and external building facades (cladding PV systems) [1]. About half of the current BIPV applications are realized in façades and one third on roofs [2]. However, to date, BIPV still remains a niche market with limited turnover [2-4].

Several drawbacks inhibit the rapid development of BIPV into a mainstream technology. By applying wireless power transfer (WPT), some of these disadvantages could be solved. In this work, we present the possible advantages of applying WPT to BIPV. We highlight the different challenges, and propose possible solutions. As far as we know, this is the first work that discusses the application of WPT into BIPV. 


\section{ADVANTAGES OF WPT IN BIPV}

A building-integrated solar module, whether it takes the form of a window, or a roof shingle, is located at the exterior of the building envelope. It contributes to realizing the physical barrier between the unconditioned outside and the conditioned inside environment. It protects the inside climate of the building against unwanted water, heat, light, noise or air. Unfortunately, a prerequisite for any BIPV system is the wired electrical connectivity from the solar panel to the inside of the building. This implies two consequences.

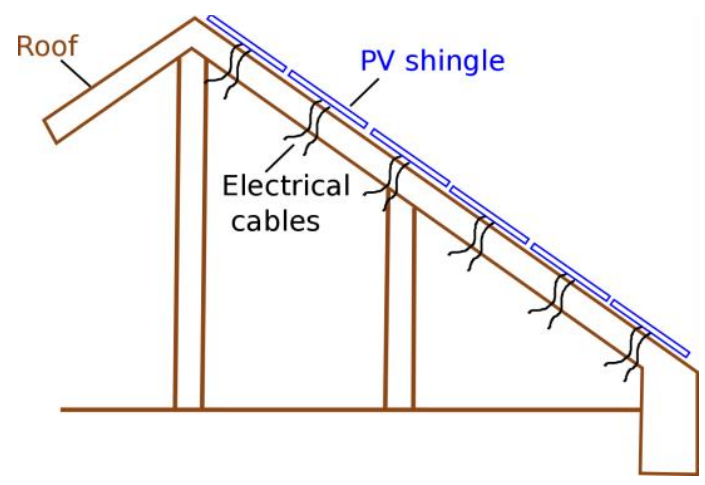

Fig. 1. Schematic overview of BIPV roof shingles: electric cables breach the thermal envelope of the building

First, the electric cables are breaking the thermal envelope of the building by the creation of thermal bridges. This reduces the building insulation and creates unwanted heat loss or gain. As an example, consider Fig. 1 which shows a representation of BIPV roof shingles. The separate shingles are connected with each other (possibly in different strings) by electric wires in the interior of the building. Each separate shingle breaches the thermal envelope by its wires. For every $10 \mathrm{~m}^{2}$ of roof area, this corresponds with about 150 breaches (a typical BIPV shingle's dimension is $184 \mathrm{~mm}$ x $365 \mathrm{~mm}$ [5]). These thermal bridges impact the energy requirements to heat or cool the building and can result in thermal discomfort. Notice that thermal bridges are not restricted to roof shingles, but apply to all BIPV applications, for example, the frame of a window covered with semitransparent solar cells will have to be pierced for the electric connections.

Second, the perforation of the electric cables through the insulation potentially causes condensation and water penetration. This results in moisture intrusion, as well for the building as for the BIPV installation, leading to a faster degradation of the PV cells, and thus a shorter lifetime of the BIPV system [6, 7].

By installing a WPT system, the produced energy from the solar modules can be transferred wirelessly over the building envelope, without the creation of any physical piercings. In this way, a nearly perfect thermal, air, and water tightness can be created, resulting in a better conditioned indoor environment, longer lifetime of the total system, and reduced cost. 
Moreover, BIPV systems with WPT could allow for an easy "plug-and-play" mechanism for installation. Nowadays, discussions arise over who is responsible for the BIPV construction elements: the construction worker or the electrician. In practice, construction workers are now educated to correctly handle the different wiring schemes of WPT. A plug-and-play system would facilitate the set-up of the system.

Aside from the plug-and-play and improved insulation, WPT would also contribute to solving the problem of reduced efficiency caused by, a.o., partial shading [8]. We elaborate this matter in the following section.

\section{CHALLENGES FOR THE PRACTICAL IMPLEMENTATION OF A WPT SYSTEM IN BIPV}

We now discuss the challenges for implementing a WPT design into BIPV. The first question that arises is the choice of the WPT technology. A promising technique that has already entered as well the consumer as the industrial market is inductive wireless power transfer (IPT). This near-field technique uses a varying magnetic field to transfer energy from a transmitter coil to a coupled receiver coil. Another near-field technique is capacitive wireless power transfer (CPT) that uses the electric field to transfer energy from a transmitter plate to a receiver plate. However, CPT has not yet significantly entered the market and its technology is not as maturely developed as IPT. Moreover, in general, the operating frequency for CPT is higher than for IPT, in the range of several MHz. For these reasons, an IPT system is preferable. The losses due to the inductive WPT link itself will be limited, on the one hand because an almost perfect alignment is possible between the transmitter and receiver coil, and on the other hand because the distance between both coils can often be restricted to a few $\mathrm{mm}$.

Photovoltaic solar cells generate DC, which has to be converted to AC for connection to the electrical grid. This conversion can occur at several locations [8, 9]. For a centralized configuration, the PV modules are connected in series and parallel arrays and the DC/AC conversion is realized by a single central inverter (Fig. 2a). For a string (Fig. 2b) or multi string (Fig. 2c) configuration, each series string of PV modules has its own DC/AC or DC/DC inverter, respectively. None of these configurations are applicable to WPT, since a prerequisite of any near-field WPT system requires AC. In order to transfer the DC electricity generated at the solar modules wirelessly, it has to be converted to AC at the solar panel itself. Therefore, a micro inverter topology is preferable (Fig. 2d) since the DC is transformed to $\mathrm{AC}$ at each separate module itself.

A second issue is the difference in frequency necessary for WPT and the frequency of the electrical grid. For WPT, frequencies in the range from $100 \mathrm{kHz}$ to several $\mathrm{MHz}$ apply, thus much higher than the $50 / 60 \mathrm{~Hz}$ of the grid. This implies the addition of an extra micro inverter to the system. Fig. 3 gives a 
schematic overview of the necessary conversions, regardless of which electromagnetic WPT technology applied. First, the DC is converted to high frequency AC in order to allow the WPT. After the wireless transfer, the high frequency AC is either converted to low frequency AC to distribute to the grid, or to DC again (which can be converted to low frequency AC to connect to the grid). All these conversions result in efficiency losses (e.g., the Enphase M215 micro inverter converts DC to AC with an efficiency of $96.5 \%[10])$.

(a)

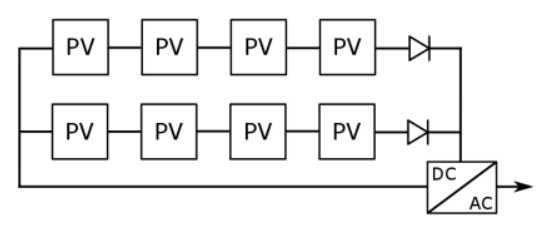

(c)

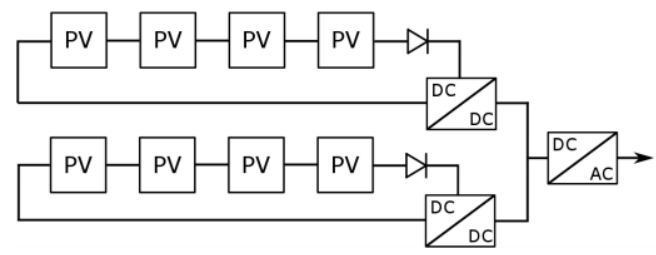

(b)

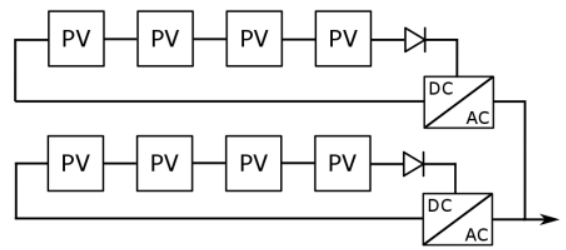

(d)

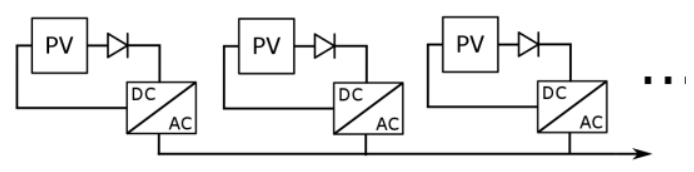

Fig. 2. (a) Centralized configuration (b) String configuration (c) Multi string configuration (d) Micro inverter configuration

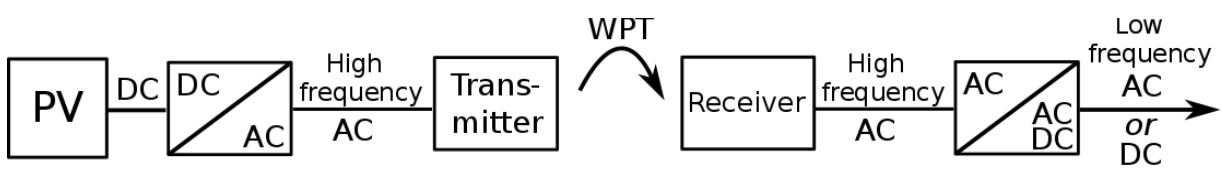

Fig. 3. Schematic overview of the necessary frequency conversions for a WPT system implemented in BIPV

There is another reason a micro inverter topology is preferable: if WPT is ever to enter the market, it will only be possible if the WPT system is compatible with current systems. For example, a BIPV setup should be able to modularly accept as well a photovoltaic window implemented with WPT, as a traditional photovoltaic window without WPT. This implies the development of a modular WPT system, integrated into the BIPV component itself.

A modular inverter lowers the efficiency and increases the cost of the system, but it has an important advantage which might warrant its use. It contributes to solving the problem of reduced efficiency caused by partial shading or module mismatching [8]. Indeed, a photovoltaic plant requires maximum power point tracking to optimize energy capture. However, if parts of the solar modules are shaded, not equally illuminated, or showing different performance (e.g., due to ageing phenomena), the location of the maximum power points on the current-voltage characteristics of the different modules is different. It the 
inverter is centralized, and not modular implemented per module, this results in a significant energy loss. A micro inverter system increases the energy-yield in turn reducing the payback time of the installation, especially for a multifaceted BIPV setup. It also protects the entire plant for malfunctioning solar modules: whereas one defective module can significantly influence the yield for a centralized or (multi)string topology, a micro inverter topology avoids this problem.

DC/AC inverters are inherently large devices and it is not always straightforward to have enough space available to implement them into an existing BIPV solution, especially with regard to the height. Typically, the total height of the micro inverter, including casing, should be limited to $40 \mathrm{~mm}$ in order to fit into the frame. However, if the structure is designed from the start with WPT in mind, it should be possible to provide the necessary room for the WPT system. For example, [11] developed a micro invertor for BIPV applications with dimensions $127 \mathrm{~mm}$ x $76 \mathrm{~mm}$, and a very limited height of only 3 $\mathrm{mm}$. The micro inverter operates at high frequency and at power levels that are required for BIPV applications.

A non-negligible heat is produced by the converters. Thus, the reliability of the electronics might also be an issue, in particular since also the solar cells achieve high temperatures. This can lead to a faster degradation, while on the other hand, the value of electronic components is temperature dependent. For example, a variable temperature changes the value of the resonator capacitance, and thus the resonant frequency of the WPT circuit. However, a dynamic tuning mechanism [12] can solve this issue. The reliability can be increased by adding sensors (temperature, voltage,...) to monitor the system and dynamically adjust its performance. In other words, it will be necessary to integrate a self-regulating solution for each module. In certain applications, cooling can be achieved by introducing air flow [4] or by passive cooling, e.g., the frame of a curtain wall or BIPV window.

\section{CONCLUSIONS}

Applying WPT to BIPV has distinct advantages, such as the reduction of thermal bridges and moisture intrusion. However, it also introduces novel challenges. To date, there is no scientific literature that describes this application. In this work, we performed a first step by listing the advantages and challenges, providing different solutions. Among others, we proposed applying a micro inverter topology with IPT as WPT technology. Implementing modular micro inverters will not only increase the compatibility for market integration, but also contribute to solving the problem of reduced efficiency due to partial shading or module mismatching. 


\section{REFERENCES}

[1] Heinstein, P., Ballif, C. and Perret-Aebi, L.E., 2013. Building integrated photovoltaics (BIPV): review, potentials, barriers and myths. Green, 3(2), pp. 125-156.

[2] Tabakovic, M., Fechner, H., Van Sark, W., Louwen, A., Georghiou, G., Makrides, G., Loucaidou, E., Ioannidou, M., Weiss, I., Arancon, S. and Betz, S., 2017. Status and outlook for building integrated photovoltaics (BIPV) in relation to educational needs in the BIPV sector. Energy Procedia, 111, pp. 993-999.

[3] Biyik, E., Araz, M., Hepbasli, A., Shahrestani, M., Yao, R., Shao, L., Essah, E., Oliveira, A.C., del Caño, T., Rico, E. and Lechón, J.L., 2017. A key review of building integrated photovoltaic (BIPV) systems. Engineering Science and Technology, an International Journal,20(3), pp. 833-858.

[4] Jelle, B.P., 2015. Building integrated photovoltaics: A concise description of the current state of the art and possible research pathways. Energies, 9(1), pp. 21.

[5] 'Tesla Solar Roof, 2018. [Online]. Available: https://www.tesla.com/solarroof. [Accessed: 10- Jan- 2018].

[6] Quintana, M.A., King, D.L., McMahon, T.J. and Osterwald, C.R., 2002, May. Commonly observed degradation in field-aged photovoltaic modules. 29th IEEE Photovoltaic Specialists Conference 2002, pp. 1436-1439.

[7] Yang, R.J., 2015. Overcoming technical barriers and risks in the application of building integrated photovoltaics (BIPV): hardware and software strategies. Automation in Construction, 51, pp. 92-102.

[8] Ikkurti, H.P. and Saha, S., 2015. A comprehensive techno-economic review of microinverters for Building Integrated Photovoltaics (BIPV). Renewable and Sustainable Energy Reviews, 47, pp. 997-1006.

[9] Liu, B., Duan, S. and Cai, T., 2011. Photovoltaic DC-building-module-based BIPV system - Concept and design considerations. IEEE Transactions on Power Electronics, 26(5), pp. 1418-1429.

[10] Enphase, ‘Enphase M215 Microinverter’, datasheet, Nov. 2016.

[11] Erickson, R.W. and Rogers, A.P., 2009, February. A microinverter for building-integrated photovoltaics. In Applied Power Electronics Conference and Exposition. 24th Annual IEEE APEC 2009, pp. 911-917.

[12] Qiang, H., Huang, X., Tan, L., Ji, Q. and Zhao, J., 2012. Achieving maximum power transfer of inductively coupled wireless power transfer system based on dynamic tuning control. Science China Technological Sciences, pp.1-8. 